\title{
Reconstruction of PC Display from Common-Mode Noise Emitting on Electrical Power Line
}

\author{
Triet Nguyen-Van ${ }^{1, *(1)}$ and Masakatsu Senda ${ }^{2}$ \\ 1 Department of Technology Management for Innovation, The University of Tokyo, Tokyo 113-8656, Japan \\ 2 Department of Innovative Electrical and Electronic Engineering, Oyama National College of Technology, \\ Oyama 323-0806, Japan; senda@oyama-ct.ac.jp \\ * Correspondence: nguyen@tmi.t.u-tokyo.ac.jp; Tel.: +81-3-5841-1599
}

Received: 3 April 2019; Accepted: 3 June 2019; Published: 6 June 2019

\begin{abstract}
This paper presents a method for reconstruction of a personal computer (PC) display image from common-mode noises coupling with monitor signals on a PC power cable. While the signal cable, which connects the PC and the monitor, is usually near the user, the power cable is connected to the outside electrical network of the office or the building. Thus, the power cables may become dominant gateways and/or antennas for emission and conduction of the common-mode noise, which may lead to a serious security issue. The measured common-mode was found to include both the monitor signal and undesired beats, which were caused by step responses of the signal and conceal the meaningful information. The original monitor signal was then calculated by excluding the beats, which could be measured by using standard up-step and down-step responses, from the measured common-mode noise and using an inverse function of the noise current level. The experimental results show that the beats were removed almost completely from the noise waveform for a monochromatic image. Alphabetic character strings, each of which were composed of, at most, $9 \times 9$ dots, were confirmed to be reconstructed clearly both in the monitor resolutions of $800 \times 600$ pixels and $1280 \times 1024$ pixels from the common-mode noise.
\end{abstract}

Keywords: common-mode noise; electromagnetic compatibility; information security; PC display; step response; power cable

\section{Introduction}

With developments of multimedia telecommunication networks, increased speed of information processing and power saving are required for networks and telecommunication equipment, including personal computers (PCs). To this purpose, various high-speed and low-voltage operation techniques have been developed and applied in many devices [1-3]. However, high-speed and low-voltage operations in communication equipment bring an undesired electromagnetic emission to other equipment and cause malfunction trouble by emission from other equipment, respectively [4-6]. Therefore, regulations [7] and countermeasure techniques [8,9] have been investigated and studied for electromagnetic compatibility (EMC) between the equipment. Emission and conduction of electromagnetic noise are also significant problems in the field of information leakage and tapping. Information reconstruction techniques from electromagnetic noise have been studied from the viewpoint of information security [10-13]. Display images on PC monitors are particularly important for security because they may contain a lot of secret and personal information [14-16]. The common-mode noise containing the information displayed on the monitor seems to appear not only from the signal cable but also from the power cable of the monitor. While the signal cable, which connects the PC and the monitor, is usually near the users, the power cable is connected to the outside electrical network of the office or the building. Thus, the power cables may become dominant gateways and/or antennas for 
noise emission and conduction, which may lead to a serious security issue. Studies on noise emitting from the power cables of the PC and its applications are not only important for research that may be used in military technology, but also contribute in increasing the awareness of the users and the device makers regarding information security issues. In this work, the distributions of common-mode noise due to the PC monitor signal are examined for the power cables of a PC and a monitor and for the monitor signal cable between the PC and the monitor. Then, we propose an algorithm for signal processing to reconstruct small size characters displayed on the monitor from common-mode noise on the power cable.

The organization of this paper is as follows: Section 2 presents the measurement system set-up for the common-mode noise of the power line. Examination of the common-mode noise and the proposed signal processing method for reconstruction of the PC display are presented in Section 3 with the discussions. Section 4 shows the experimental results for the elimination of undesired beats and reconstruction of a character string from the common-mode noise. Conclusions are presented in Section 5.

\section{Measurement System Set-Up}

The measurement set-up for the common-mode noise emitting from the power cables is shown in Figure 1. A desktop PC and a progressive cathode-ray tube (CRT) monitor were either put on a wooden table with a height of $0.8 \mathrm{~m}$ or on the floor. A liquid crystal monitor (LCD) was also examined as a monitor, but no difference between CRT and LCD was observed for the noise measurement. This work used a CRT monitor because its resolution can be set flexibly in analog, unlike an LCD, whose resolution is restricted digitally by the number of image cells. The power cables of the PC and the monitor were independently connected to outlets on the wall of a laboratory, which was on the second floor in a ferroconcrete building. An analog Video Graphics Array (VGA) cable carrying analog components RGBHV (red, green, blue, horizontal synchronization, vertical synchronization) was used as a monitor signal cable connected between the PC and the monitor. The coaxial and the parallel type of power cables were connected in series for measuring both common-mode and normal-mode currents. Cable sheaths, shielding, and ferrite cores of these power cables and signal cables were removed in order to measure a normal-mode current and to facilitate measurement of small noise current. A split-core active current probe (A6302, Tektronix, US) with an amplifier (AM503, Tektronix, US) whose $3 \mathrm{~dB}$ bandwidth was at $50 \mathrm{MHz}$ was used for detecting the current on the cables. The output from the current probe was measured by using a spectrum analyzer or a digital oscilloscope. A total $3 \mathrm{~dB}$ bandwidth of this measurement system was confirmed to be $50 \mathrm{MHz}$. The serial data of the noise current derived from the oscilloscope was then used for the signal processing. A specification of the monitor signal was set mainly at a resolution of $800 \times 600$ pixels and a refresh rate of $60 \mathrm{~Hz}$, where a period of vertical synchronizing signal was $16 \mathrm{~ms}$ $(60 \mathrm{~Hz})$, a period of horizontal synchronizing signal was $26 \mu \mathrm{s}(38 \mathrm{kHz})$, and a dot clock frequency was $40 \mathrm{MHz}(25 \mathrm{~ns})$. Another resolution of $1280 \times 1024$ pixels was also tested to examine the performance of the signal processing method proposed in this work.

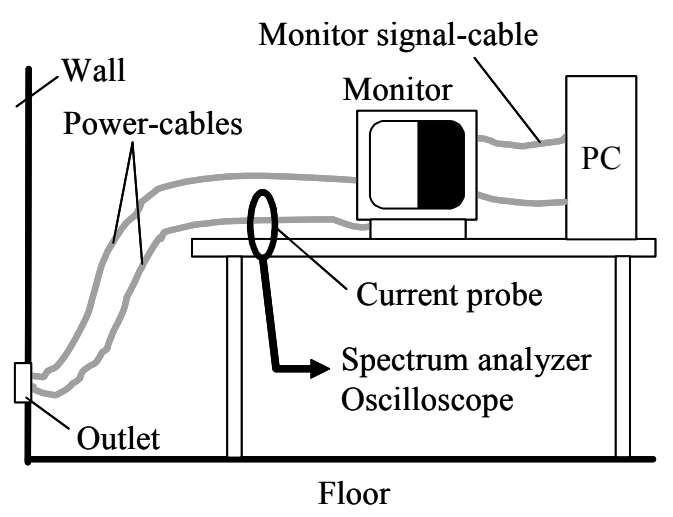

Figure 1. Measurement set-up for noise on cables. 


\section{Common-Mode Noise and Signal Processing Method for Reconstruction of PC Display}

\subsection{Measurement of Common-Mode Noise}

In a desktop PC, the power cables are connected to the electrical outlets, which may extend outdoors even if the PC stands alone. Thus, we first examined the distribution of the common-mode noise caused by the monitor signal on the PC and the monitor power cables to detect the possibility of remote information reconstruction. The experiments were carried out for both cases, where the power cables were put on the table and on the floor. Each power cable had the length of $1.85 \mathrm{~m}$. A coaxial parallel serial cable with a length of $1.85 \mathrm{~m}$ was used for the monitor signal cable.

Figure 2 shows the level of the common-mode noises depending on the measurement position, which was defined by the distance along with the power cables from the outlet to the current probe used for the measurement. The noise levels were estimated as peak to peak current values measured with the current probe and averaged three times. A simple image in which the left half was the white color and the right half was the black color (shown in Figure 1) was displayed on the monitor with a resolution of $800 \times 600$ pixels. The noise level was measured at a fundamental frequency of $38 \mathrm{kHz}$ with a spectrum analyzer. The noise levels were too low to observe with the oscilloscope. At the probe positions of more than $1.6 \mathrm{~m}$ (near the PC or the monitor), the noise levels became high except for the case where the PC power cable was put on the floor. In the cases where the position of the current probe was less than $1.6 \mathrm{~m}$, the levels exhibited roughly constant values of $20-40 \mu \mathrm{A}$. The constant noise levels were reasonable if the common-mode noise current was caused by the capacitive coupling between the cables and the earth [17].
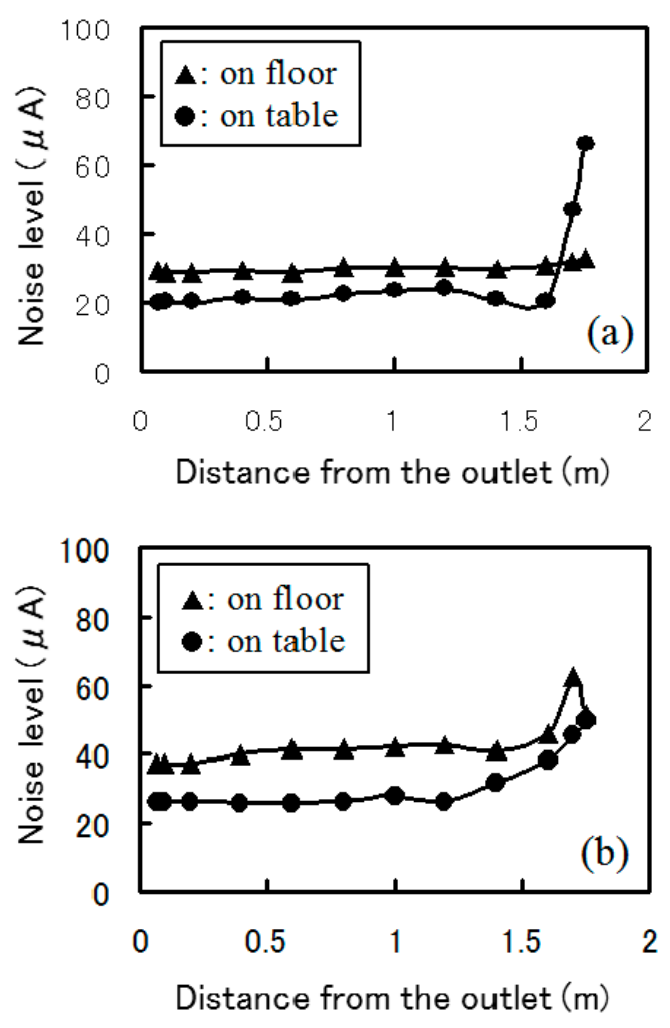

Figure 2. Dependences of the common-mode noise level on the distance from the outlet of the power cables: (a) personal computer (PC) power cable, (b) monitor power cable.

Figure 3 shows the common-mode noise distribution on the monitor signal cable under the same measurement conditions as above. In this case, the position was defined as the distance along the signal cable from the PC to the current probe. In Figure 3a, the levels were measured only on the coaxial part of the serial cable. The levels were constantly independent of the position, and there was 
no difference observed between the cases when the signal cable was put on the table and on the floor. The common-mode noise levels measured with only the coaxial cable for a monitor signal cable are shown in Figure $3 \mathrm{~b}$ for a comparison. The noise levels decreased to about one-third compared with that of the coaxial parallel series cable shown in Figure 3a; this was a result of the noise reduction effect by the coaxial cable.
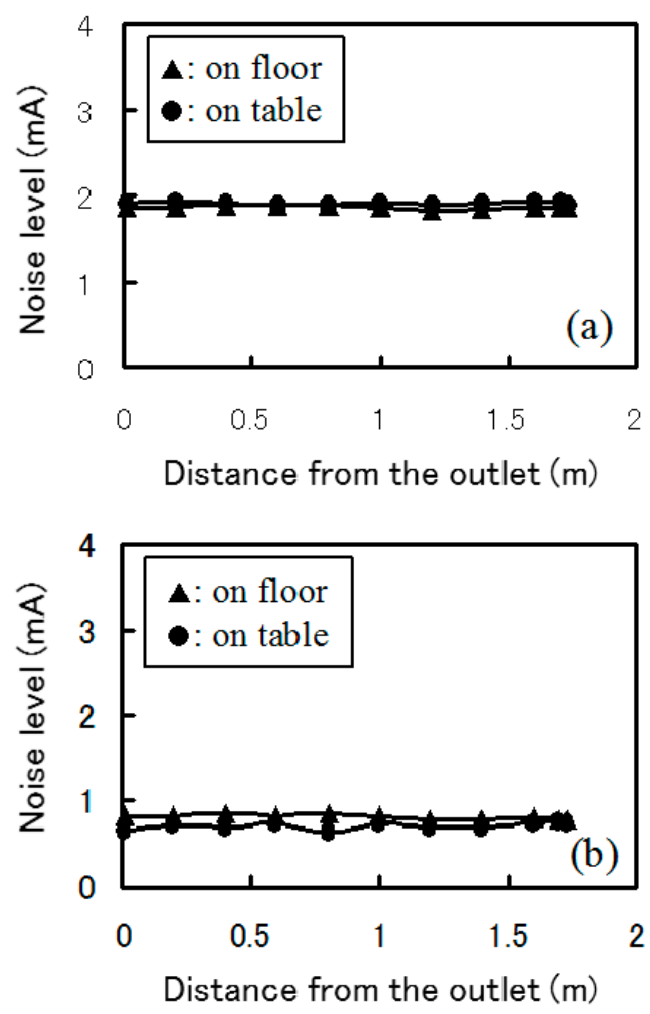

Figure 3. Dependences of the common-mode noise level on the distance from the monitor of the signal cables: (a) coaxial parallel series cable, $(\mathbf{b})$ only coaxial cable.

From the above experimental results, we can confirm that the common-mode noise, due to monitor signal, conducts on power cables and leaks toward the outdoor. The current level of the noise measured with this system was too low for waveform-observation, so the noise on monitor signal-cable was aimed for reconstruction, as mentioned in the next section.

\subsection{Reconstruction of PC Display-Image}

\subsubsection{Examination on Undesired Beats Accompanying with the Common-Mode Noise}

The smallest characters that could be displayed on a PC monitor of the measurement system without any defects were the alphabetic characters with the size of $9 \times 9$ dots. It is very meaningful in information security to reproduce these characters from the common-mode noise. Thus, we set these characters as the target objects for reconstruction.

At first, three one-dot vertical lines (black/white/black/white/black lines on a white background) were displayed on the monitor to examine the response of common-mode noise for a standard display image. The waveforms for the normal-mode and the common-mode noise currents are shown in Figure 4 . The observed display images are shown in the insets. The coaxial parallel series cable was used for the signal cable, and the red color signal line was selectively probed at a parallel part for the normal-mode and at a coaxial part for the common-mode, respectively. The monitor was set at a resolution of $800 \times 600$ pixels. The normal-mode or the common-mode pulse wave due to the vertical synchronizing signal was used as a trigger for the oscilloscope. In the normal-mode, 
the noise current value changed by several $\mathrm{mA}$ between high (corresponding to the white color) and low (corresponding to the black color) levels. In this case, the one-dot width corresponded to $25 \mathrm{~ns}$ (40 MHz). Dull responses for the up- and the down-steps may have been caused by the bandwidth of $50 \mathrm{MHz}$ in this measurement system. The normal-mode waveform was regarded to be almost equal to the monitor signal except for the dullness. On the other hand, the change reduced to about $2 \mathrm{~mA}$, and the waveform was accompanied by complicated beats in the common-mode. Such beats were not observed for the reference signals from a pulse generator with the same current probe and oscilloscope used in this measurement. Therefore, these beats were interpreted to be generated intrinsically from the measured PC system itself.
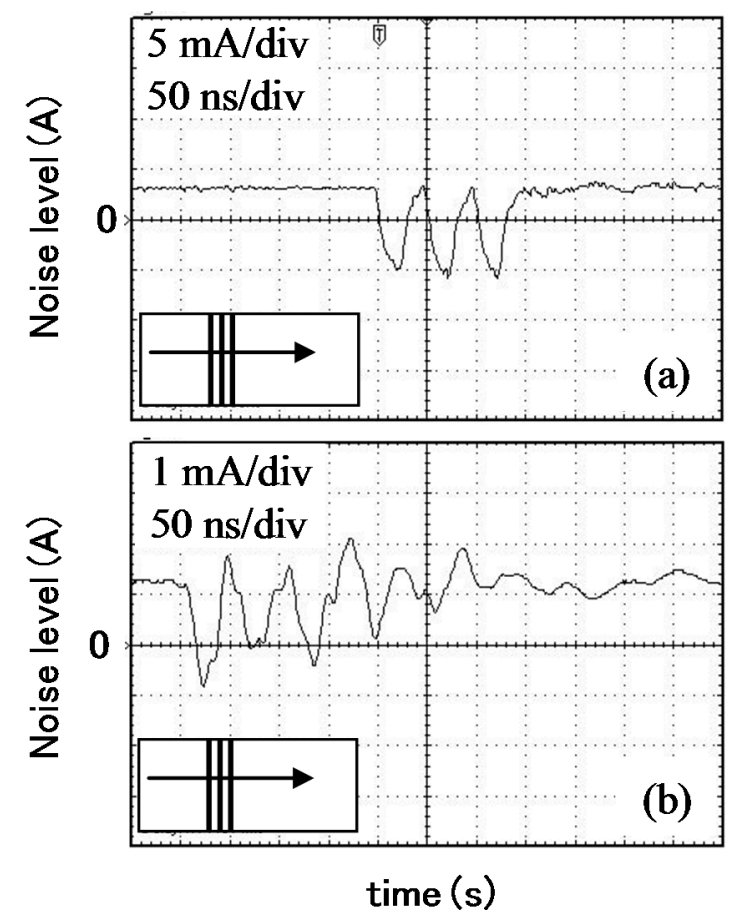

Figure 4. Waveforms of the noise level for: (a) normal-mode, (b) common-mode.

The above beats needed to be removed from the common-mode noise waveform to reconstruct the display images with a high resolution of one-dot. Thus, in the next step, we examined details of the beats by analyzing the responses for the up- and the down-step signals. A half-white and half-black image, as shown in Figure 1, was used for up- or down-step signals. The up- and the down-step responses were upper and lower symmetry. The beats seemed to appear only just after the steps where the display images changed steeply, as shown in Figure 5. This indicated that the beats were generated as step responses by a quadratic transfer function, which was caused by the electromagnetic induction and the capacitive coupling in the PC system.

Remark 1. The superimposed beats accompanying the common-mode noise for the image, where the up-step and the down-step repeat continuously (horizontal dot-line), affect the common-mode noise waveform most seriously. The mentioned above three one-dot vertical lines are a simple example of such images. 


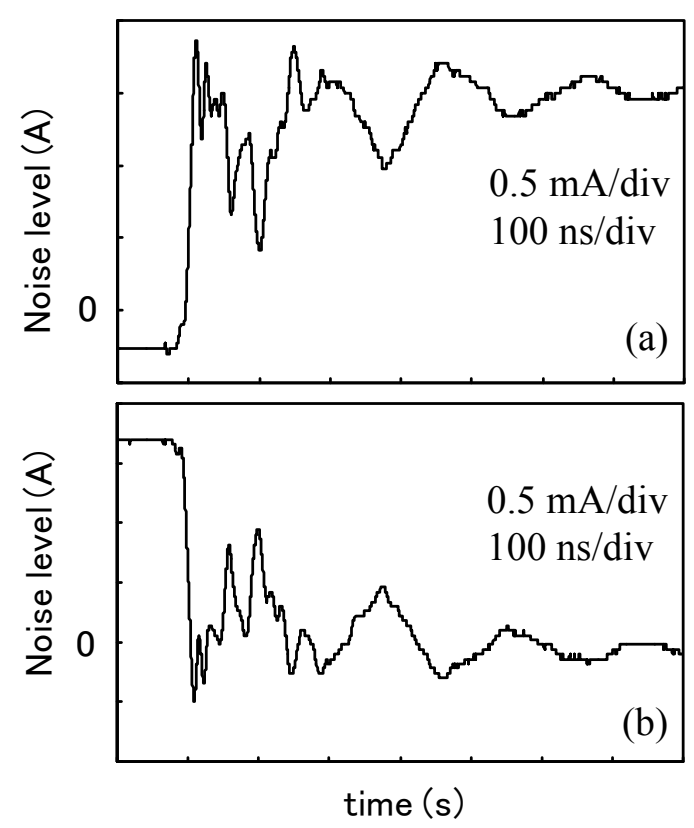

Figure 5. Step responses of the common-mode noise for: (a) up-step, (b) down-step.

\subsubsection{Signal Processing Method for Reconstruction of PC Displayed Image}

Let $f$ be a monitor signal and $g$ be a corresponding common-mode noise. If we can obtain a mathematical expression of the transfer function, the monitor signal $f$ may be reconstructed analytically from the common-mode noise $g$. Let $F, F_{s}, G$, and $G_{s}$ be the Laplace transforms of the monitor signal $f$, the step signal $f_{s}$, the measured response $g$, and the step response $g_{s}$, respectively. Then, we have the following relation:

$$
F=\frac{F_{s}}{G_{s}} G
$$

While the transfer functions $G_{s}$ and $F_{s}$ are known, and the function $G$ is measurable, the function $F$ can be calculated by using the relation given by Equation (1). Then, the monitor signal $f$ is calculated by using the inverse Laplace transform of the function $F$ as:

$$
f=\mathcal{L}^{-1}(F)=\mathcal{L}^{-1}\left(\frac{F_{s}}{G_{s}} G\right)
$$

In this study, we tried to solve the above inverse transferring with numerical analysis by using the actual measured numerical data.

Let the reference monitor signal and the measured current of the common-mode noise at an instant $k$ be $u(k)$ and $i(k)$, respectively. The measured current can be written by:

$$
i(k)=I(u(k))+\sum_{i=0}^{k} N_{i}(k)
$$

where $I(u(k))$ is the noise current level of the reference signal $u(k)$, and $N_{i}(k)$ is the value of the beat accompanied with the step change of the reference signal that occurs at instant $i$. The beat $N_{i}$ can be written by:

$$
N_{i}=\left\{\begin{array}{cl}
N_{u p} & \text { if } u(i)-u(i-1)>0 \\
0 & \text { if } u(i)-u(i-1)=0 \\
N_{\text {down }} & \text { if } u(i)-u(i-1)<0
\end{array}\right.
$$

where $N_{u p}$ and $N_{\text {down }}$ are the beats caused by the up-step and the down-step of the reference signal, which are measurable and are shown by Figure 5 . 
Thus, the reference signal $u$ can be reconstructed by using Equation (3) as:

$$
u(k)=I^{-1}\left[i(k)-\sum_{i=0}^{k} N_{i}(k)\right]
$$

where $I^{-1}$ is the inversion of the noise current level $I$.

Figure 6 shows the sequential frame of the information writing on the monitor. The signal for each line in the monitor started by a horizontal synchronizing signal and moved sequentially. The low signal level continued until reaching the display area. In the display area, the monitor signal exhibited a high or a low level corresponding to the white or the black color. In this study, the dot clock frequency of the monitor was assumed to be known and was used for synchronizing the measured data to the monitor image line by line.

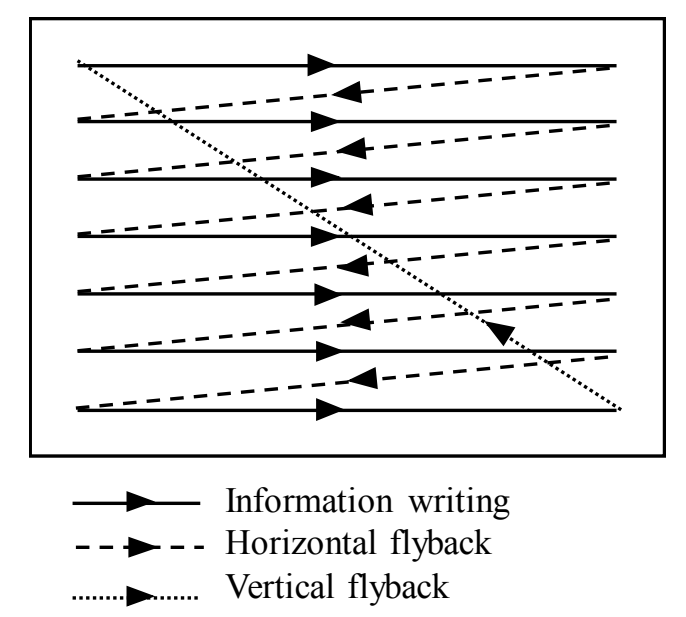

Figure 6. Sequential frame of the information writing on the monitor.

The following algorithm was proposed for the signal processing to remove the beats from the measured current. At the beginning, the monitor signal was assumed to be high level (white color) just after entering the display area. The common-mode noise level was also high. The noise level was observed when it decreased continuously-for example, by ten times. The level was judged to change from high to low if it did. Then, the down-step response shown in Figure $5 b$ was subtracted numerically from the original noise waveform. The beginning point for the subtraction corresponded to the instant when the noise level began to decrease in the above judgment. At this instant, the common-mode noise level was low, and the change from low to high level was judged by a continuous increase of the level. The up-step response shown in Figure 5a was subtracted numerically from the above waveform processed for the first down-step. After this, similar processes were repeated.

The dot clock frequency was given as a characteristic property of the monitor. The timing when the noise level should have been judged for continuous increase, decrease, or no change could be recognized accurately by an elapsed time from a common-mode pulse wave due to the horizontal synchronizing signal. Thus, the noise waveforms could be processed more accurately in this case.

\section{Experimental Results}

\subsection{Elimination of Undesired Beat from Common-Mode Noise for the Three One-Dot Vertical Lines}

The proposed signal processing was applied to eliminate the undesired beat from the common-mode noise for the three one-dot vertical lines described in Section 3.2.1. The same image for the three one-dot vertical lines as is shown in the inset of Figure 4 was displayed on the monitor. The measurement setup is presented in Section 2. Figure 7 shows the changes in the common-mode noise waveforms by the above 
signal processing. Figure 7a shows an original noise waveform before the processing. The waveform was reformed (as shown in Figure $7 \mathrm{~b}$ ) after processing for the first down-step. Then, the waveform shown in Figure $7 \mathrm{~b}$ was reformed to the waveform shown in Figure $7 \mathrm{c}$ after processing for the first up-step. Afterwards, the waveforms continued to be processed similarly. Finally, the beats were eliminated almost completely, and only three sharp pulses could be derived, as shown in Figure 7g.
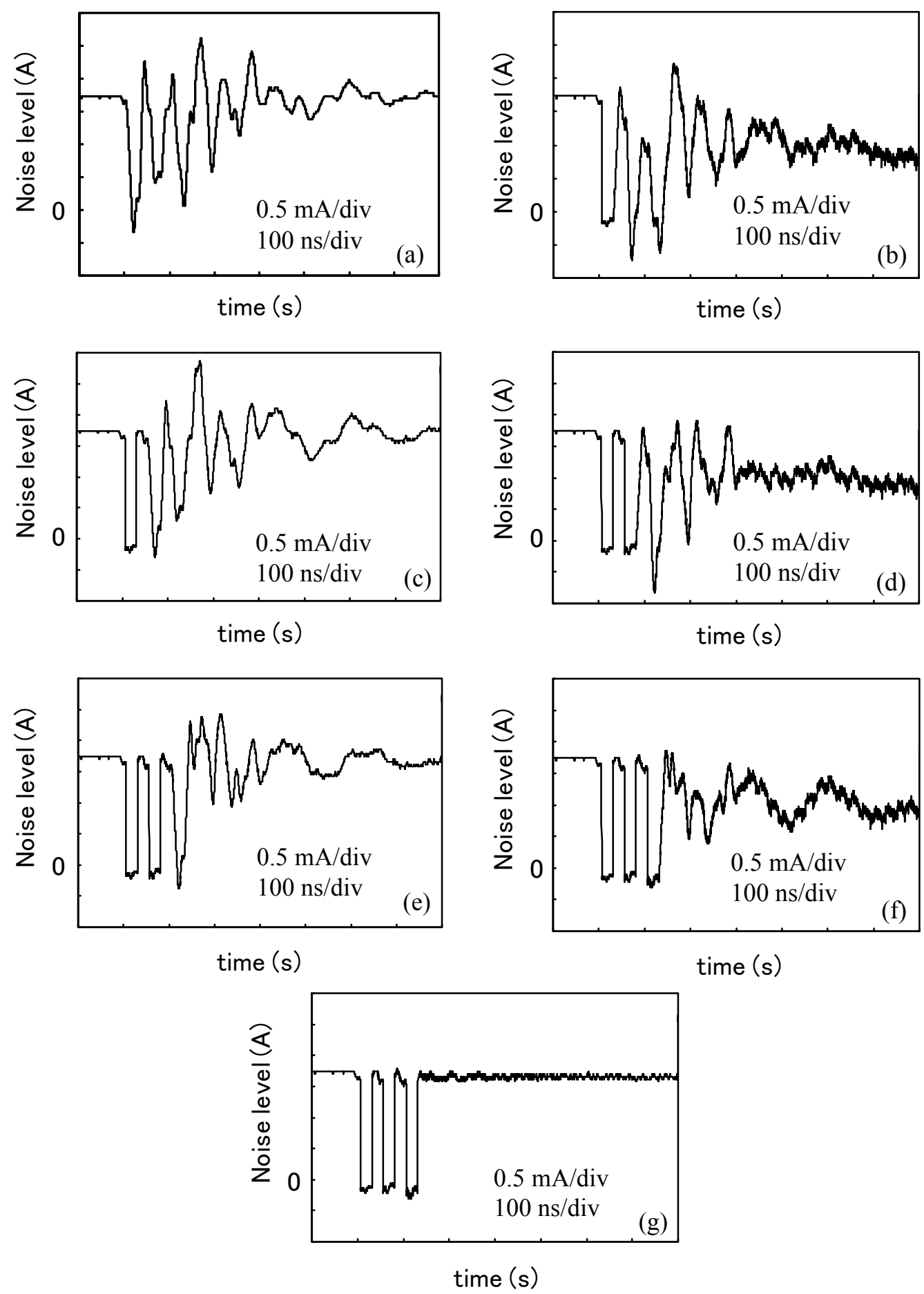

Figure 7. Common-mode noise waveforms of the signal processing: (a) before processing, (b) after processing for first down-step, (c) after processing for first up-step, (d) after processing for second down-step, (e) after processing for second up-step, (f) after processing for third down-step, (g) after processing for third up-step. 


\subsection{Reconstruction of a Character String}

The above proposed signal processing was applied to reconstruct the character string "EMC", which was written with Tahoma font and was displayed on the monitor, as shown in Figure 8. The character string was displayed monochromatically with black dots on a white background. Each character was composed of, at most, $9 \times 9$ dots in which one dot corresponded to one pixel of the $800 \times 600$ pixels resolution of the monitor. The common-mode noise waveform for the top horizontal line of the characters is shown in Figure 9. By using the proposed signal processing, the waveform shown in Figure 9a was reformed to the image in Figure 9b, displaying shaped pulse responses without any beat. Figure 10 shows the reproduced image expressed in 8-bit gradation after the whole processing. We confirmed that the characters were reproduced clearly from the measured common-mode noise emitting from the power cable. The small ripples on high and low levels in the waveform shown in Figure $9 \mathrm{~b}$ produced the slight gray parts shown in Figure 10. A clearer image could be obtained by using an additionally simple binary signal processing with a threshold level.

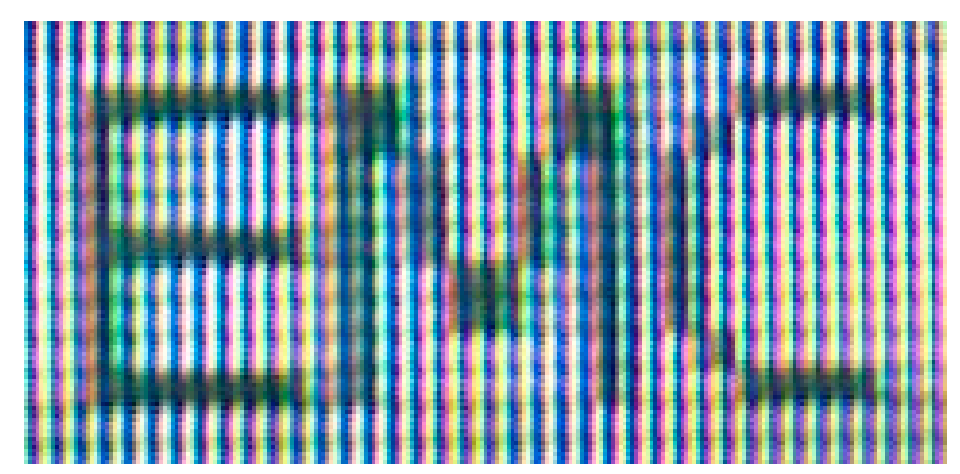

Figure 8. Character string displayed on monitor $(800 \times 600$ pixels $)$.

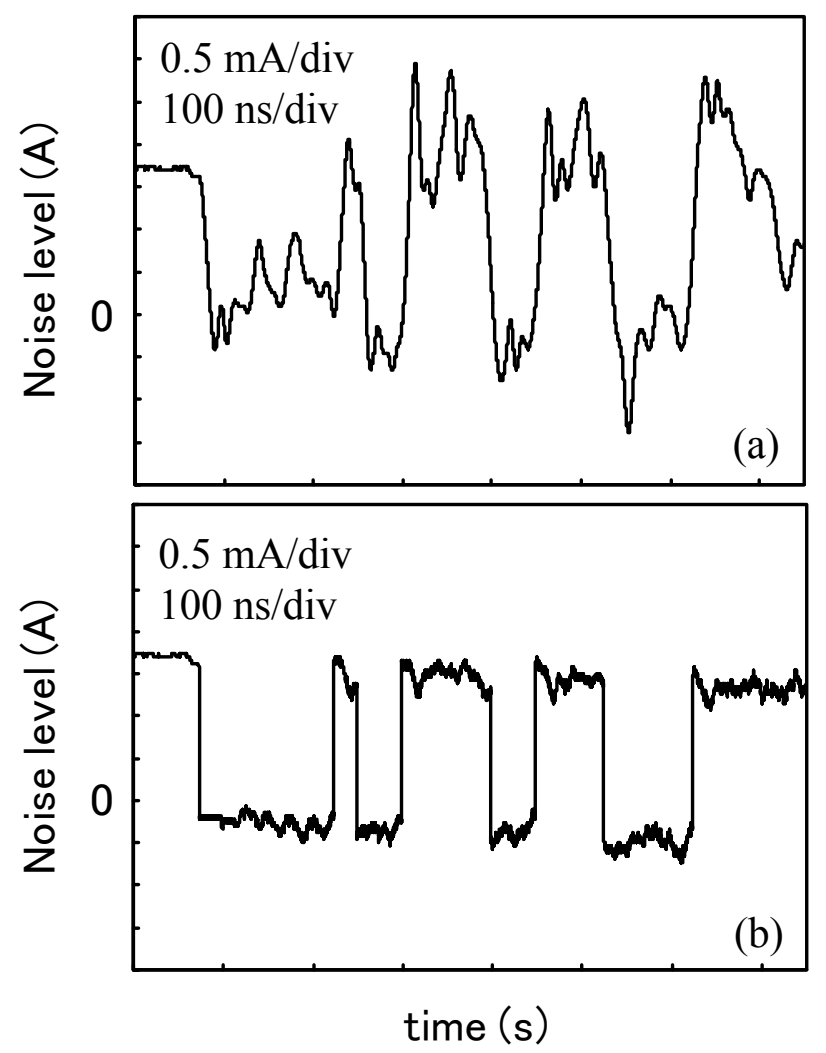

Figure 9. Common-mode noise waveforms for $800 \times 600$ pixels monitor specification: (a) before processing, (b) after processing. 


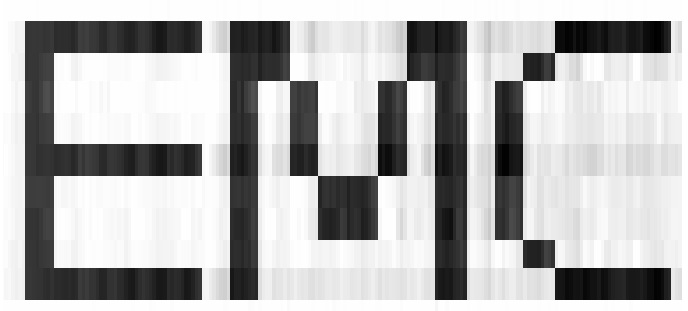

Figure 10. Reconstructed image from common-mode noise $800 \times 600$ pixels monitor specification.

For further testing, the proposed reconstruction method was tested for an image with the resolution of $1280 \times 1024$ pixels and the dot clock frequency of $110 \mathrm{MHz}$. The same character string, "EMC", as shown in Figure 11, was selected as the target image. The common-mode noise waveforms before and after the signal processing for the top horizontal line of the character string are shown in Figure 12a,b, respectively. Shaped pulse responses similar to those in Figure $9 \mathrm{~b}$ could be derived, despite the fact that the dot clock frequency of $110 \mathrm{MHz}$ was higher than the measurement bandwidth of $50 \mathrm{MHz}$. Figure 13 shows the reconstructed image, where the character string composed of (at most) $9 \times 9$ dots displayed in the $1280 \times 1024$ pixels monitor specification was reconstructed successfully.

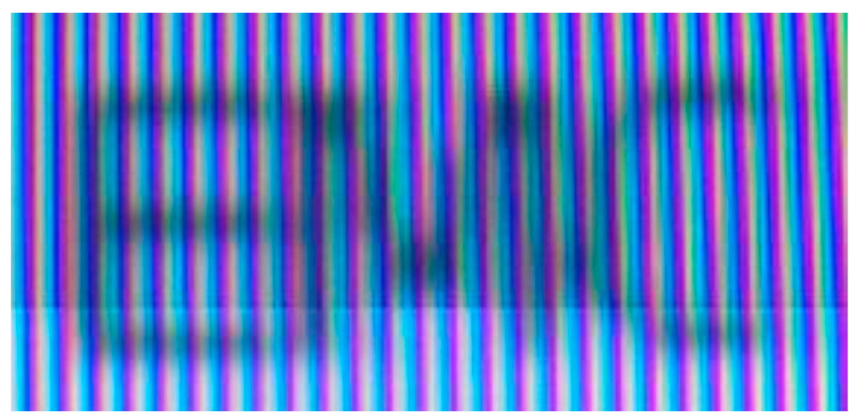

Figure 11. Character string displayed on monitor $(1280 \times 1024$ pixels $)$.
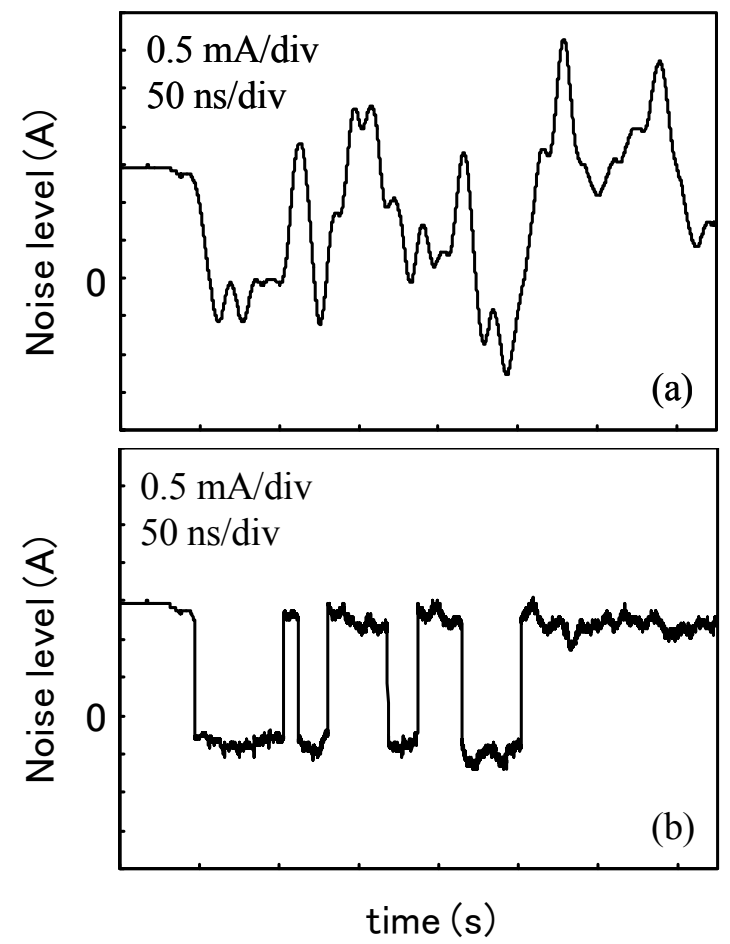

Figure 12. Common-mode noise waveforms for $1280 \times 1024$ pixels monitor specification: (a) before processing, (b) after processing. 


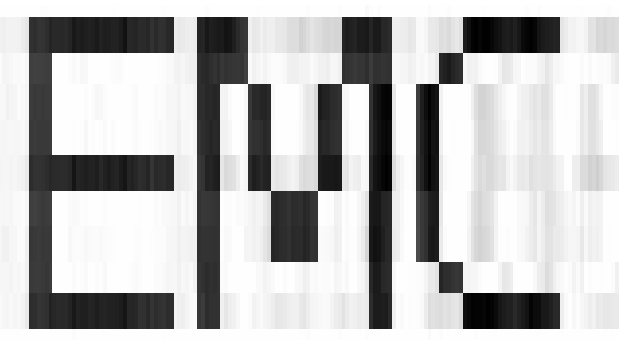

Figure 13. Reconstructed image from common-mode noise for $1280 \times 1024$ pixels monitor specification.

Remark 2. In the proposed signal processing, the dot clock frequency of the monitor was assumed to be known and was used for synchronizing the measured data to the monitor image line by line. In the case where this frequency is unknown, the proposed processing algorithm needs to be tuned until it meets the appropriate frequency.

Remark 3. It may require a more complex signal processing to reconstruct a multicolor image due to separated responses of red, green, and blue signals.

\section{Conclusions}

In this study, a signal processing algorithm was proposed to reconstruct a PC display image from the common-mode noise emitting on the PC power cable, which was easily measurable by using the current probe. The complicated beats, which were caused by the up-step and the down-step responses of the signal, concealed the meaningful information included in the common-mode noise. The proposed signal processing algorithm was used to exclude the beats from the measured common-mode noise and to reconstruct the original monitor signal. An alphabetic character string composed of, at most, $9 \times 9$ dots was reproduced clearly from common-mode noise for both cases, where the monitor resolutions of $800 \times 600$ and $1280 \times 1024$ pixels were used. Although this work was carried out under the condition of removing shielding and filters from cables and only for monochromatic images, the results indicate that nearly fine images and a character string displayed on the monitor could be reconstructed relatively easily with appropriate signal processing as long as the common-mode noise could be detected. Although the experiments were carried out for the simple character string, the proposed method may be extended for reconstructing more complex images or motion scenes in real-time processing. While the Internet of Things (IoT) technology is being used in a wide range of industry and usual life, and the number of IoT devices is increasing day by day, such security issues as those mentioned in this paper should be considered more seriously.

Author Contributions: Conceptualization, M.S.; methodology, M.S. and T.N.-V.; measurement, T.N.-V.; analysis and validation, M.S. and T.N.-V., programming, T.N.-V.; writing-original draft preparation, T.N.-V.; writing-review and editing, M.S. and T.N.-V.; supervision, M.S.

Conflicts of Interest: The authors declare no conflict of interest.

\section{References}

1. Xu, D.; Xu, S.; Chen, G. High-speed low-power and low-power supply voltage dynamic comparator. Electron. Lett. 2015, 51, 1914-1916. [CrossRef]

2. Nasr, B.; Wang, D.; Kruk, R.; Rösner, H.; Hahn, H.; Dasgupta, S. High-Speed, Low-Voltage, and Environmentally Stable Operation of Electrochemically Gated Zinc Oxide Nanowire Field-Effect Transistors. Adv. Funct. Mater. 2013, 23, 1750-1758. [CrossRef]

3. Neag, M.; McCarthy, O. High speed, low-power CMOS voltage buffers. In Proceedings of the International Semiconductor Conference, Sinaia, Romania, 6-10 October 1998; pp. 175-180. 
4. Bisognin, P.; Pelissou, P.; Cissou, R.; Giniaux, M.; Vargas, O. High speed link radiated emission reduction. In Proceedings of the ESA Workshop on Aerospace EMC, Valencia, Spain, 23-25 May 2016; pp. 1-4.

5. Wu, T.L.; Lin, Y.H.; Wang, T.K.; Wang, C.C.; Chen, S.T. Electromagnetic bandgap power/ground planes for wideband suppression of ground bounce noise and radiated emission in high-speed circuits. IEEE Trans. Microw. Theory Tech. 2005, 53, 2935-2942.

6. Stocksreiter, W.; List, H.; Berger, H.; Weis, G.; Krenn, M.; Engel, G. Electromagnetic Emissions in High Density and Fast GaN Switched Half Bridges with Resonance Filter Structures. In Proceedings of the International Exhibition and Conference for Power Electronics, Intelligent Motion, Renewable Energy and Energy Management, Nuremberg, Germany, 10-12 May 2016; pp. 2151-2158.

7. Amemiya, F.; Hattori, M. Overview of international standardization on electromagnetic immunity requirements for telecommunications equipment. NTT Rev. 1997, 9, 14-19.

8. Senda, M.; Ishii, O.; Mori, T. Application to an EMI noise filter of a high-loss $\mathrm{CoZrNb} / \mathrm{SiO}_{2}$ multilayer film. IEEE Transl. J. Magn. Jpn. 1994, 9, 78-83. [CrossRef]

9. Mori, T.; Senda, M. EMI noise reduction tape containing magnetic-alloy film. IEICE Trans. Commun. 2000, E83-B, 600-607.

10. Eck, M.V. Electromagnetic radiation from video display units: An eavesdropping risk? Comput. Secur. 1985, 4, 269-286.

11. Kuhn, M.G.; Anderson, R.J. Soft tempest: Hidden data transmission using electromagnetic emanations. In Information Hiding 1998, Lecture Notes in Computer Science; Springer: Berlin, Germany, 1998; Volume 1525, pp. 200-210.

12. Kuhn, M.G. Optical time-domain eavesdropping risks of CRT displays. In Proceedings of the IEEE Symposium on Security and Piracy, Berkeley, CA, USA, 12-15 May 2002; pp. 3-18.

13. Sekiguchi, H.; Tanaka, H.; Seto, S.; Yamamura, A. A study of the reconstruction of display image on a PC's monitor by unintentional electromagnetic emanation, emanated electromagnetic signal measurement method and reconstructed display image using measured signal emanation. IEICE Tech. Rep. 2005, ISEC2005-97, 53-58.

14. Yuichi, H.; Naofumi, H.; Mamoru, M.; Takafumi, A.; Hideaki, S. A Threat for Tablet PCs in Public Space: Remote Visualization of Screen Images Using EM Emanation. In Proceedings of the ACM SIGSAC Conference on Computer and Communications Security, Scottsdale, AZ, USA, 3-7 November 2014; pp. 954-965.

15. Yasunao, S.; Masao, M.; Kimihiro, T. Countermeasure Technique for Preventing Information Leakage Caused by Unintentional PC Display Emanations. In Proceedings of the International Symposium on EMC, Kyoto, Japan, 20-24 July 2009; pp. 9-12.

16. Song, T.L.; Jeong, Y.R.; Yook, J.G. Modeling of leaked digital video signal and information recovery rate as a function of SNR. IEEE Trans. Electromagn. Compat. 2015, 57, 164-172. [CrossRef]

17. Ideguchi, K.; Koga, H.; Shimoshio, Y.; Ueda, N. Electromagnetic Noise Problem and Countermeasure Techniques; Morikita: Tokyo, Japan, 1997; pp. 29-30. 